\title{
Análise da percepção dos professores em relação aos sentimentos dos alunos em sala de aula*
}

\author{
PROFESSORS' PERCEPTION OF STUDENTS' FEELINGS IN THE CLASSROOM: AN \\ ANALYSIS
}

\author{
ANÁLISIS DE LA PERCEPCIÓN DE LOS PROFESORES EN RELACIÓN A LOS \\ SENTIMIENTOS DE LOS ALUMNOS EN SALA DE CLASES
}

Aline Raquel Sgariboldi ${ }^{1}$, Ana Cláudia Giesbrecht Puggina ${ }^{2}$, Maria Júlia Paes da Silva ${ }^{3}$

\section{RESUMO}

O estudo teve como objetivo verificar a diferença na percepção dos sentimentos dos alunos pelos professores antes e depois de uma apresentação explicativa sobre o tema. Estudo de natureza exploratória de fonte primária, abordagem quantitativa com 13 professores de Enfermagem e Medicina. Um vídeo com a imagem dos alunos foi apresentado aos professores que responderam ao questionário de identificação de sentimentos e, após uma apresentação explicativa sobre comunicação não verbal e sentimentos, assistiram ao mesmo vídeo e responderam novamente ao questionário. Quanto à identificação de sentimentos, a alegria, a ansiedade e o interesse foram os mais identificados. Encontramos valores estatisticamente significantes em relação à média da pontuação total antes e depois da apresentação explicativa $(p=0,02)$. O professor é capaz de identificar os sentimentos, porém, após a apresentação explicativa, sua percepção melhorou e ele conseguiu identificá-los mais vezes.

\section{DESCRITORES}

Estudantes

Docentes

Comunicação não-verbal

Emoções

Percepção

\begin{abstract}
The objective of this study is to verify the difference in professors' perception of students' feelings before and after an explanatory presentation on the theme. This is an exploratory study using a primary source, and a quantitative approach with 13 Nursing and Medicine professors. A vide showing student images was presented to the professors, who answered a feeling-identification questionnaire, and, after an explanatory presentation about non-verbal communication and feelings, the professors watched the same video and answered the questionnaire again. As to the identification of feelings, joy, anxiety, and interest were the most often identified. We found statistically significant values regarding the mean total score before and after the explanatory presentation $(p=0.02)$. The professor is $\mathrm{ca}$ pable of identifying the feelings, but after the explanatory presentation, their perception improved and they were able to make identifications more often.
\end{abstract}

DESCRIPTORS
Students
Faculty
Nonverbal communication
Emotions
Perception

\section{RESUMEN}

El estudio objetivó verificar la diferencia en la percepción de los sentimientos de los alumnos por los profesores antes y después de una presentación explicativa sobre el tema. Estudio exploratorio de fuente primaria, abordaje cuantitativo con 13 profesores de Enfermería y Medicina. Un video con la imagen de los alumnos se presentó a los profesores que respondieron el cuestionario de identificación de sentimientos y, luego de una presentación explicativa sobre comunicación no-verbal y sentimientos, vieron el mismo video y respondieron nuevamente el cuestionario. Respecto de identificación de sentimientos, alegría ansiedad e interés fueron los más identificados. Encontramos valores estadísticamente significativos en relación a la media de puntuación total antes y después de la presentación explicativa $(p=0,02)$. El profesor es capaz de identificar los sentimientos, sin embargo luego de la presentación explicativa, su percepción mejoró y le fue posible identificar sentimientos mayor cantidad de veces.

\author{
DESCRIPTORES \\ Estudiantes \\ Docentes \\ Comunicación no verbal \\ Emociones \\ Percepción
}

\footnotetext{
* Extraído da monografia "Análise da percepção dos professores em relação aos sentimentos dos alunos em sala de aula", Conclusão de Curso de Enfermagem da Faculdade de Medicina de Jundiaí, 2008. ${ }^{1}$ Enfermeira Graduada pela Faculdade de Medicina de Jundiaí. Jundiaí, SP, Brasil. aline_fmj@yahoo.com.br ${ }^{2}$ Enfermeira. Doutoranda da Escola de Enfermagem da Universidade de São Paulo. Professora Assistente da Faculdade de Medicina de Jundiaí. Membro do Grupo Estudo e Pesquisa sobre Comunicação em Enfermagem da Escola de Enfermagem da Universidade de São Paulo. São Paulo, SP, Brasil. claudiapuggina@usp.br ${ }^{3}$ Enfermeira. Professora Titular do Departamento de Enfermagem Médico-Cirúrgica da Escola de Enfermagem da Universidade de São Paulo. Diretora do Departamento de Enfermagem do Hospital Universitário da Universidade de São Paulo. Coordenadora do Grupo Estudo e Pesquisa sobre Comunicação em Enfermagem. São Paulo, SP, Brasil. juliaps@usp.br
} 


\section{INTRODUÇÃO}

A palavra comunicar, etimologicamente, origina-se do latim communicare, que significa pôr em comum ${ }^{(1)}$. A comunicação define-se como uma forma de compreender e compartilhar mensagens enviadas e recebidas. Para este ato, devem estar presentes o emissor, o receptor, a mensagem, o canal e a resposta ${ }^{(2)}$.

A comunicação interpessoal pode ser dividida em comunicação verbal e não-verbal. A comunicação verbal está associada às palavras expressas por meio da linguagem escrita ou falada e a comunicação não-verbal àquela que envolve todas as manifestações de comportamento não expressas diretamente por palavras. A comunicação não-verbal é a informação obtida por meio de posturas, expressões faciais, gestos, orientações do corpo, singularidades somáticas, distância mantida entre os indivíduos e até mesmo a organização dos objetos no espaço ${ }^{(3)}$.

Segundo estudos de psicologia social, estima-se que apenas 7\% dos pensamentos são expressos por palavras, $38 \%$ são por sinais paralinguísticos (entonação da voz, velocidade das palavras ditas, entre outros) e $55 \%$ são por sinais do corpo, ou seja, não-verbal ${ }^{(3)}$.

Os sinais não-verbais fornecem vários significados e apresentam quatro funções principais no processo de comunicação interpessoal, sendo elas: complementar à comunicação verbal, substituir a comunicação verbal, contradizer o verbal e demonstrar sentimentos. Demonstrar sentimentos significa demonstrar qualquer emoção que não seja apenas por palavras, mas principalmente por meio das expressões faciais ${ }^{(3)}$.

Uma pessoa perceptiva ou intuitiva refere-se àquela que possui capacidade de ler os sinais de linguagem corporal das outras pessoas e compará-los aos sinais verbais, identificando desta forma as contradições ${ }^{(4)}$.

A emoção humana é a ativação de uma rede neural complexa e elaborada que promove um repertório bastante variado de respostas comportamentais ${ }^{(5)}$. O sentimento é definido como a faculdade ou a capacidade de sentir e de receber impressões mentais. Além disso, é considerado um conjunto de emoções ${ }^{(6)}$.

A face do ser humano é capaz de demonstrar emoções, sendo considerada um meio de comunicação e tradução de praticamente todas as reações ou emoções ${ }^{(7-8)}$. Presume-se que somos capazes de fazer e reconhecer cerca de 250 mil expressões faciais( ${ }^{(9)}$. Existe um resumo das descrições de expressões faciais de emoções ${ }^{(8,10-11)}$.

Reconhecer essas expressões faciais pode ser fundamental para que o professor elabore intervenções e meIhore o aprendizado dos alunos.
Ensinar é estimular para a identificação e resolução de problemas, é ajudar a criar novos hábitos de pensamento e de ação ${ }^{(12)}$. Ensinar é também interagir, aproximar e fazer dialogar dois universos diferentes ${ }^{(13)}$.

Há autores que consideram a principal virtude do professor a capacidade que ele tem para transmitir conhecimentos. Porém, a emissão, transmissão e recepção de informação é apenas uma das funções da comunicação entre professor e aluno ${ }^{(12)}$.

O professor precisa despertar a atenção e interesse, mobilizar a inteligência do aluno, ser entendido por este e induzi-lo à expressão e ao diálogo, e não estar somente preocupado em expor sua matéria; ou seja, comunicar-se adequadamente ${ }^{(12)}$. É necessário que ele trabalhe vários aspectos do aluno, como a sua afetividade, suas percepções, sua expressão, seus sentidos, suas críticas, sua criatividade ${ }^{(8)}$.

Além disso, existem comportamentos comunicativos verbais e não-verbais que podem motivar o aluno a aprender. A motivação é uma condição interna do indivíduo e, no aprendizado, os sentimentos, as relações interpessoais, a afetividade, as diferenças culturais, as crenças e os valores estão envolvidos ${ }^{(14)}$.

Na área da educação, é utilizado o recurso de devolutiva que é uma informação fornecida ao aluno sobre o seu desempenho em uma determinada situação ou atividade ${ }^{(15)}$. No momento em que ocorre essa devolutiva a comunicação não-verbal é muito importante, visto que é necessário que o professor interprete e valide a comunicação não-verbal do aluno. Se esta validação não ocorrer, a interpretação pode ser distorcida e a mudança no padrão de desempenho não ocorrer, impedindo desta maneira o processo de aprendizagem.

Alguns dos sinais não-verbais que podem ser observados no aluno em sala de aula, durante o processo de aprendizagem, são: postura corporal, localização na sala de aula, contato dos olhos, roupas, expressão facial, volume de voz, distância interpessoal mantida, toque e movimentação da cabeça ${ }^{(3)}$.

Em uma aula teórica, a compreensão é mais evidente quando há interação entre professor e aluno, existindo neste momento uma troca constante de vários níveis de comunicação. A interação com os alunos envolve a percepção do comportamento do professor e dos alunos, para que se possa ajustar a aula de acordo com a reação detectada e intervir de diversas maneiras para garantir a motivação e aumentar a retenção ${ }^{(16)}$.

A universidade, por sua vez, tem o compromisso de preparar e conscientizar o professor sobre o seu papel de comunicador para que o ensino seja flexível e atual ${ }^{(17)}$. Alguns 
professores possuem um limite pessoal maior, comparado a outros, em desenvolver a percepção para identificar os sentimentos dos alunos durante as aulas. Uma das hipóteses para que isto ocorra é quando ele está iniciando a carreira na docência e possivelmente por estar preocupado em transmitir as informações e conteúdos das aulas. Além disso, há instituições de ensino superior que não oferecem ao professor condições para que os sentimentos sejam identificados, pois existem salas de aulas que contêm um grande número de alunos e estrutura física inadequada.

A inspiração para a realização desta pesquisa surgiu do interesse no tema comunicação não-verbal, sua relação com o processo de ensino-aprendizagem e em verificar se os professores de uma instituição privada de ensino superior conhecem o que ela é e se são capazes de identificar os sentimentos dos alunos em uma filmagem dos mesmos.

A aplicação prática dessa pesquisa é atentar professores e instituições de ensino sobre os benefícios de um educador que utiliza e sabe identificar a comunicação não-verbal dos alunos durante o processo de ensino-aprendizagem e que essa percepção pode e deve ser treinada através de cursos, leituras e dinâmicas sobre o tema comunicação.

\section{OBJETIVO}

Verificar qual a diferença na percepção dos sentimentos dos alunos pelos professores antes e depois de uma apresentação explicativa sobre o tema, no contexto da educação superior.

\section{MÉTODO}

Tipo de estudo: estudo de natureza exploratória de fonte primária, por se tratar de pesquisa de campo com abordagem quantitativa.

Local do estudo: uma Autarquia Municipal de Ensino Superior do Município de Jundiaí.

Amostra: foram convidados a participar da pesquisa todos os professores que ministram aulas no curso de Enfermagem e Medicina, pois esta instituição é formada por estes cursos. Contudo, a amostra foi constituída de 13 professores, sendo sete professores do curso de Enfermagem e seis professores do curso de Medicina.

Critérios de inclusão: ser docente da instituição do estudo e ter no mínimo um ano de docência. Esse tempo mínimo foi estabelecido porque, possivelmente, com a experiência o professor não estaria somente preocupado em ministrar o conteúdo, mas em identificar as necessidades e reações dos alunos.

Comitê de Ética em Pesquisa: o Projeto de Pesquisa foi submetido ao Comitê de Ética em Pesquisa da Faculdade de Medicina de Jundiaí e, após a aprovação, foi iniciado (Protocolo FMJ 129/08). O Termo de Consentimento Livre e Esclarecido foi entregue aos pesquisados e foram considerados sujeitos da pesquisa aqueles que leram, concordaram e assinaram o mesmo em duas vias.

\section{Preparação do material}

Etapa 1 - Vídeo de apresentação para os alunos: foi elaborada uma apresentação para os alunos com vídeos obtidos da internet com duração de 2'56", contendo propagandas de diversos contextos com a intenção de desencadear diferentes reações em um curto período de tempo. As propagandas continham cenas engraçadas, suaves e trágicas.

Etapa 2 - Questionário de identificação de sentimentos: foi elaborado um questionário direcionado aos professores onde deveriam assinalar se identificaram ou não os sentimentos citados (alegria, ansiedade, cansaço, desinteresse, dúvida, interesse, medo, raiva, surpresa e tristeza) e, no caso de resposta afirmativa, descreveriam como os identificaram. Optou-se por solicitar que os professores descrevessem os sentimentos identificados para garantir que os itens não fossem simplesmente assinalados sem a reflexão do professor.

Etapa 3 - Questionário de caracterização de amostra: foi elaborado um questionário contendo nove questões sobre a identificação pessoal (sexo, idade, estado civil, profissão, formação acadêmica e tempo de atuação como professor) e o tema da pesquisa (comunicação não-verbal, processo de aprendizagem e percepção dos sentimentos).

Etapa 4 - Apresentação explicativa: foi elaborada uma apresentação em PowerPoint com 12 slides, direcionada aos professores, contendo a definição dos tipos de comunicação (verbal e não-verbal), as funções da comunicação não-verbal, explicações sobre os sentimentos com figuras para uma melhor compreensão e exemplos de alguns sinais que podem ser observados no aluno em sala de aula. Esta apresentação foi apreciada por um pesquisador em comunicação não-verbal, atuando este como juiz de adequação do conteúdo.

Etapa 5 - Filmagem dos alunos: os vídeos de propaganda foram apresentados a um grupo de cinco alunos filmados em uma disposição convencional de sala de aula. Estes alunos foram convidados aleatoriamente para participar da filmagem e são conhecidos pelos sujeitos da pesquisa. Definiu-se essa quantidade de pessoas porque a imagem captada pela filmagem é menor comparada com a imagem real (ao vivo) e, sendo um grupo menor, permitiria uma aproximação para melhor identificação dos sentimentos. A filmadora ficou posicionada em um local estratégico, garantindo que todos os sujeitos da pesquisa fossem filmados e que a presença de um objeto diferente na sala de aula não dispersaria a atenção do vídeo que estava sendo apresentado.

\section{Procedimentos para a coleta de dados}

Os professores foram convidados por e-mail a participar da pesquisa e agendado um dia para a coleta de da- 
dos, de acordo com a disponibilidade e sua permanência na instituição. A coleta de dados foi iniciada e as seguintes etapas foram seguidas:

Etapa 1 - Apresentação da filmagem dos alunos aos professores: o professor não foi informado a princípio sobre os objetivos da pesquisa (mascarado para o pesquisado) para evitar que ele fosse influenciado a prestar atenção nos sentimentos dos alunos.

Etapa 2 - Preenchimento do questionário de identificação de sentimentos antes da apresentação explicativa: o professor foi orientado a assinalar quantas vezes e de que forma identificou os sentimentos.

Etapa 3 - Preenchimento do questionário de caracterização da amostra que continha um total nove questões, sendo seis questões sobre identificação pessoal e três questões sobre o tema da pesquisa.

Etapa 4 - Apresentação explicativa em PowerPoint: a apresentação explicativa foi mostrada em forma de slides pela pesquisadora e, logo depois, foi entregue uma cópia da mesma ao professor. Esta apresentação teve uma duração em média de 10 minutos.

Etapa 5-2a apresentação da filmagem dos alunos aos professores: o professor assistiu novamente a mesma filmagem dos alunos.

Etapa 6 - Preenchimento do questionário de identificação de sentimentos depois da apresentação explicativa: o professor novamente deveria assinalar quantas vezes e de que forma identificou os sentimentos.

Etapa 7 - Devolutiva: ao término da coleta de dados, foi apresentada ao professor a mesma filmagem, porém com legendas dos possíveis sentimentos.

O tempo de coleta de dados com cada professor foi de aproximadamente 30 minutos.

\section{Tratamento dos dados}

Os dados foram armazenados no Excel for Windows Explorer $^{\circledR}$ e analisados no Software SAS (Statistical Analysis System) versão 9.01 por um profissional especializado. Para a obtenção dos resultados, foram utilizados os seguintes testes:

- Teste T de Student Pareado para a comparação das médias;

- Teste de Qui-quadrado de McNemar para a comparação dos percentuais.

A significância estatística dos testes foi avaliada de acordo com o padrão da literatura geralmente aceitável e o $p$ do teste foi considerado estatisticamente significativo se $p<0,05^{(18)}$.

Para a realização da análise dos dados do questionário de identificação de sentimentos, foi utilizado um sistema de pontuação, onde as respostas foram classificadas em sim ou não. Sendo assim, caso fosse assinalado sim, a pontuação era somada (maior que zero) e caso fosse assinalado não, a pontuação não era somada (igual a zero). Contudo, foi obtida a média do número de professores que marcaram em cada um dos sentimentos. Além disso, foi realizada uma comparação das médias antes e depois da apresentação explicativa.

\section{RESULTADOS}

Foram convidados a participar do estudo 36 professores, porém, apenas 14 compuseram a amostragem inicial. Um dos sujeitos da pesquisa foi excluído devido ao não preenchimento correto dos questionários. A amostra constituiu-se, portanto, de 13 professores. Os demais professores não compareceram para a realização da coleta de dados e, por tratar-se de uma pesquisa destinada à monografia de conclusão de curso de graduação, não foi possível realizar um novo agendamento com os professores.

Os 13 professores que participaram da pesquisa tinham em média 44,8 anos ( $\pm 10,7$ desvio-padrão), idade mínima de 27 anos e máxima de 61 anos. A amostra foi composta predominantemente por pessoas do sexo feminino (9-69,2\%).

Em relação ao estado civil a maioria dos professores eram casados $(9-69,2 \%)$, os outros solteiros $(3-13$; $23,1 \%$ ) e separado $(1-7,7 \%)$. Quanto à profissão $7(53,9 \%)$ eram enfermeiros, 3 (23\%) eram médicos e $3(23 \%)$ de outras profissões (cirurgião dentista, pedagoga e biomédica).

Todos os professores (100\%) possuíam pós-graduação, sendo que $6(46,1 \%)$ possuíam doutorado, 4 (30,8\%) especialização e $3(23 \%)$ mestrado.

O tempo de atuação como professor foi em média de 15,2 anos ( $\pm 11,2$ desvio-padrão), sendo o tempo máximo de 35 anos e mínimo de 5 anos.

Em relação à comunicação não-verbal, 12 (92,3\%) dos professores responderam que têm conhecimento do tema. Relataram que já leram algum artigo científico, livro ou texto $(9-69,2 \%)$, assistiram a palestras $(7-53,8 \%)$ e realizaram cursos que abordassem o tema ( $2-15,4 \%)$. Apenas 1 (7,7\%) professor relatou não ter conhecimento sobre o tema.

Todos os professores participantes $(100 \%)$ consideraram que a identificação de sentimentos dos alunos é importante para o processo de aprendizagem.

Os professores apresentaram justificativas em relação à importância da identificação dos sentimentos dos alunos para o processo de aprendizagem, através do qual eles poderiam mudar e melhorar a estratégia de ensino. Os principais itens apresentados estavam relacionados a mudar a estratégia de ensino, facilitar o processo de aprendizagem, verificar o entendimento do aluno sobre o conteúdo da aula, seu interesse, estimular para o aprendizado e promover a interação na sala de aula. 
Sobre a expressão de sentimentos em sala de aula, todos os professores (100\%) relataram possuir lembrança de alguma situação em sala de aula em que a expressão dos sentimentos foi evidente. Nessas experiências relatadas, o sentimento de alegria foi o mais mencionado pelos professores participantes (4 - 30,8\%).

Todos os professores conseguiram exemplificar situações em que a expressão dos sentimentos foi evidente em sala de aula, como, por exemplo, a demonstração de cansaço, desinteresse, alegria, dúvida, vontade de fazer pergunta, etc.

No estudo, os sentimentos de alegria (100\%), ansiedade $(92,3 \%)$ e interesse (100\%) foram os sentimentos mais identificados. Alguns sentimentos como cansaço, desinteresse, raiva e surpresa foram mais identificados após a apresentação explicativa. O número de sujeitos que identificou os sentimentos de dúvida e medo não alterou após a explicação. Com exceção da tristeza, todos os outros sentimentos foram mais identificados após a apresentação explicativa ou não se alteraram.

Na Tabela 1 é apresentado a média e o desvio-padrão de quantas vezes cada professor identificou um sentimento antes e depois da apresentação explicativa. Para o cálculo da média de cada sentimento, foram somadas quantas vezes os professores identificaram cada sentimento e isso foi dividido pelo número de professores.

Tabela 1 - Média e desvio-padrão de quantas vezes o professor identificou cada sentimento antes e depois da apresentação explicativa - Jundiaí - 2008

\begin{tabular}{|c|c|c|c|c|c|}
\hline \multirow{2}{*}{ Sentimento } & \multicolumn{2}{|c|}{ Antes } & \multicolumn{2}{|c|}{ Depois } & \multirow{2}{*}{ valor- $p$} \\
\hline & Média & Desvio-padrão & Média & Desvio-padrão & \\
\hline Alegria & 3,0 & 1,2 & 3,2 & 1,4 & 0,2 \\
\hline Ansiedade & 1,7 & 1,2 & 1,9 & 1,3 & 0,2 \\
\hline Cansaço & 0,6 & 0,9 & 0,8 & 1,0 & 0,5 \\
\hline Desinteresse & 1,2 & 1,2 & 1,5 & 1,5 & 0,1 \\
\hline Dúvida & 1,0 & 0,9 & 1,1 & 1,0 & 0,6 \\
\hline Interesse & 1,9 & 0 & 2,1 & 1,0 & 0,2 \\
\hline Medo & 0,1 & 0,3 & 0,1 & 0,3 & 1,0 \\
\hline Raiva & 0,2 & 0,4 & 0,5 & 0,8 & 0,1 \\
\hline Surpresa & 1,0 & 1,0 & 1,1 & 0,9 & 0,8 \\
\hline Tristeza & 0,8 & 1,1 & 1,1 & 1,7 & 0,4 \\
\hline Total & 11,5 & 3,4 & 13,2 & 4,7 & 0,02 \\
\hline
\end{tabular}

Nota: Teste t de Student Pareado. A diferença foi considerada significativa se $p<0,05$.

A média de quantas vezes os professores identificaram os sentimentos após a apresentação explicativa foi maior em todos os sentimentos, com exceção do medo que não se alterou. Comparando as médias, os sentimentos identificados mais vezes pelos professores foram alegria, ansiedade e interesse.

Não foram identificadas diferenças estatisticamente significativas na comparação das médias antes e depois em cada um dos sentimentos separadamente. Porém, foi encontrada diferença estatisticamente significativa $(p=0,02)$ na comparação da soma das médias antes (média total antes) e depois da apresentação.

A média total de quantas vezes os sentimentos foram identificados, considerando todos os sentimentos, depois da apresentação explicativa foi maior (média de 13,2 e \pm 4,7 desvio-padrão) do que antes da apresentação (média de 11,5 e $\pm 3,4$ desvio-padrão).

Comparando a categoria profissional dos professores com a identificação dos sentimentos - sete enfermeiros, três médicos e três outras categorias (cirurgião dentista, pedagoga e biomédica) - verificou-se que a diferença antes e depois na percepção dos sentimentos dos alunos foi maior na categoria dos outros profissionais, pois eles con- seguiram identificar maior número de sentimentos comparados com as outras categorias profissionais enfermeiro e médico.

\section{DISCUSSÃO}

A amostra, composta predominantemente por professores do sexo feminino ( 9 - 69,2\%), pode ser um viés importante, já que as mulheres tendem a ser mais perceptivas e atentas para a linguagem corporal do que os homens. Elas possuem um olhar atento para os detalhes e uma capacidade inata de captar e decifrar os sinais não-verbais ${ }^{(4)}$.

Devido à organização cerebral da mulher, a maioria delas tem melhor desempenho comparado aos homens em relação à capacidade de comunicação. Imagens de Ressonância Magnética mostram claramente por que as mulheres têm mais capacidade de se comunicar e avaliar as pessoas do que os homens. Elas utilizam 14 a 16 áreas do cérebro para avaliar o comportamento das pessoas, contra 4 a 6 áreas no caso dos homens ${ }^{(4)}$.

Verificou-se através dos comentários dos professores durante o estudo que, além da importância da identifica- 
ção dos sentimentos em sala de aula, existe um grande interesse dos professores em aprofundar-se nesse assunto. O tempo de coleta de dados previsto para aproximadamente 30 minutos foi ultrapassado várias vezes devido aos comentários e à necessidade dos sujeitos da pesquisa em compartilhar suas experiências. No entanto, apesar de reconheceram a importância, nem todos os professores já leram algo sobre comunicação não-verbal, sendo 69,2\% aqueles que leram e uma minoria $(15,4 \%)$ que já realizou cursos que abordassem o tema.

Quando questionados sobre experiências anteriores, todos os professores relataram possuir lembrança de alguma situação em sala de aula em que a expressão dos sentimentos foi evidente; nestas a alegria $(30,8 \%)$ foi o sentimento mais mencionado.

As características faciais dos indivíduos que sorriem são mais marcantes e fáceis de serem lembradas e reconhecidas. Essa é a principal conclusão de um estudo em que os pesquisadores fotografaram 96 estudantes universitários com expressões neutras e com expressões de alegria, totalizando 192 imagens. Para as fotos com sorriso, os estudantes foram instruídos a sorrir naturalmente com a boca fechada e sem mostrar os dentes. As imagens foram divididas aleatoriamente em dois conjuntos de 96 imagens com números iguais das quatro categorias de faces (neutra masculina, sorriso masculino, neutra feminina, sorriso feminino). Os voluntários (56 homens e 58 mulheres) analisaram um desses conjuntos e tinham que classificar as faces observadas em quatro pontuações de 0 (não distinta) até três (muito distinta) pontos ${ }^{(19)}$. Faces sorridentes são mais distintas das faces neutras de forma estatisticamente significante.

Outro estudo sobre expressões faciais afirma que estas raramente são percebidas isoladamente; o reconhecimento das faces geralmente é influenciado pelo contexto em que estão envolvidas. Nesse estudo, foi solicitado aos participantes que categorizassem as expressões faciais em três tipos (aversão, medo, felicidade) mostradas em contextos com significado emocional congruente ou incongruente. Então, foi perguntado aos participantes se os contextos influenciaram o reconhecimento explícito das expressões faciais. Os resultados mostraram uma vantagem da resposta para as expressões faciais acompanhadas de contextos congruentes, ou seja, o ambiente é um fator importante no reconhecimento das expressões faciais ${ }^{(20)}$.

No estudo, a média de quantas vezes cada professor identificou um sentimento antes e depois da apresentação explicativa foi maior em todos os sentimentos, com exceção do medo que não se alterou, comprovando a hipótese de que os professores conseguiriam identificar maior número de sentimentos dos alunos após a apresentação explicativa sobre o tema.

Os sentimentos identificados mais vezes pelos professores foram alegria, ansiedade e interesse. A diferença total encontrada depois da apresentação explicativa foi significativa $(p=0,02)$.

A repetição e o treino ainda são variáveis muito importantes que devem ser levadas em consideração quando se está interessado na aprendizagem. Em um estudo, foi realizada a comparação da identificação de sinais não-verbais por enfermeiros que haviam lido algo sobre o tema e outros que não leram. Houve um aumento significativo na percepção dos sinais não-verbais dos enfermeiros que informaram ter lido algo sobre o tema. Isso demonstra que é possível aprimorar o aprendizado do não-verbal através de leitura sobre o tema ${ }^{(21)}$.

Apesar de neste estudo ter sido utilizado a apresentação explicativa para a comparação dos resultados, não tem como garantir se o professor prestou a devida atenção à mesma. Além disso, a coleta de dados foi realizada em um laboratório de internet, localizado dentro da instituição, devido à necessidade de utilização de um computador e pela facilidade de acesso a este local em qualquer período. Com isso, a escolha do local pode ter influenciado na atenção direcionada do professor ao vídeo e a apresentação explicativa, devido ao fluxo de pessoas no local.

Ao preocupar-se em ler os sinais da linguagem corporal dos alunos, o professor se tornará mais consciente e atento nas suas atividades, além de mais sensível em relação às emoções e sentimentos próprios e alheios. Isto acontece porque as emoções e os gestos têm uma estreita relação de reciprocidade $\mathrm{e}^{(3-4,8)}$.

A sala de aula como um lugar de demonstração de emoção, descontração, afetividade, respeito pelo outro, reconhecimento e valorização das diferenças individuais pode estimular a construção do conhecimento e fortalecer o vínculo professor e aluno ${ }^{(14)}$.

Esta instituição privada de ensino superior não possui salas de aula com grande número de alunos, comparada com outras instituições, visto que no curso de Medicina o número máximo é de 60 alunos e no curso de Enfermagem 40 alunos. Isto possibilita que o professor identifique os sentimentos dos alunos durante a aula. O método de ensino utilizado depende de cada professor e da disciplina a ser ministrada. Alguns professores utilizam um método ativo, no qual existe a possibilidade de interação entre professor e aluno, facilitando desta maneira a identificação dos sentimentos dos alunos. Em contrapartida, alguns professores optam pelo método expositivo limitando a interação com o aluno e, consequentemente, diminuindo a percepção dos sentimentos.

O fornecimento de devolutivas leva a uma aprendizagem próspera e domínio de conteúdo e habilidades. Este retorno é valioso para a aprendizagem, visto que ressalta as dissonâncias entre o resultado esperado e o real e incentiva a mudança. Os comportamentos adequados também são apontados por esta devolutiva, motivando a repetição do acerto $^{(22)}$. 


\section{CONCLUSÃo}

Considerando-se os resultados estatisticamente significativos, podemos concluir que existe uma diferença na identificação dos sentimentos, em geral, após a apresentação explicativa.

Percebeu-se que o professor é capaz de identificar os sentimentos dos alunos, porém, após sua atenção ter sido

\section{REFERÊNCIAS}

1. Mendes IAC. Enfoque humanístico à comunicação em enfermagem. São Paulo: Sarvier; 1994.

2. Pagliuca LMF, Fiúza NLG, Rebouças CBA. Aspectos da comunicação da enfermeira com o deficiente auditivo. Rev Esc Enferm USP. 2007;41(3):411-8.

3. Silva MJP. Comunicação tem remédio: a comunicação nas relações interpessoais em saúde. São Paulo: Loyola; 2003.

4. Pease A, Pease B. Desvendando os segredos da linguagem corporal. Rio de Janeiro: Sextante; 2005.

5. Oliveira L, Pereira MG, Volchan E. Processamento emocional no cérebro humano. In: Lent R. Neurociência da mente e do comportamento. Rio de Janeiro: Guanabara Koogan; 2008. p. 253-69.

6. Weiszflog W. Michaelis moderno dicionário da língua portuguesa. São Paulo: Melhoramentos; 2004.

7. Bueno VF, Macedo EC. Julgamento de estados emocionais em faces esquemáticas por meio da música por crianças. Psicol Teor Prat. 2004;6(2):27-36.

8. Davis F. A comunicação não-verbal. São Paulo: Summus; 1979

9. Birdwhistell RL. Kinesics and context: essays on body motion communication. Philadelphia: University of Pensylvania Press; 1970.

10. Corraze J. As comunicações não-verbais. Rio de Janeiro: Zahar; 1982.

11. Knapp ML. La comunicación no verbal: el cuerpo y el entorno. Barcelona: Paidós; 1980.

12. Bordenave JD, Pereira AM. Estratégias de ensino-aprendizagem. Petrópolis: Vozes; 2002. direcionada à comunicação não-verbal, a percepção meIhorou e ele conseguiu identificar mais vezes os sentimentos, com exceção do sentimento medo que não se alterou.

A oportunidade de compartilhar conhecimento sobre comunicação, sentimentos e o envolvimento com o ensino foi gratificante e construtivo, além de proporcionar um momento de reflexão do processo de ensino-aprendizagem para os professores.

13. Castro RK, Silva MJP. Influências do comportamento comunicativo não-verbal do docente em sala de aula: visão dos docentes de enfermagem. Rev Esc Enferm USP. 2001;35(4):381-9.

14. Rocha EM, Silva MJP. Comportamento comunicativo do docente de enfermagem e sua influência na aprendizagem do educando. Nursing (São Paulo). 2001;4(32):30-4.

15. Zeferino AMB, Domingues RCL, Amaral E. Feedback como estratégia de aprendizado no ensino médico. Rev Bras Educ Med. 2007;31(2):176-9.

16. Pazin Filho A. Interação com a platéia. Medicina (Ribeirão Preto). 2007;40(1):42-50.

17. Braga EM, Silva MJP. Comunicação competente: visão de enfermeiros especialistas em comunicação. Acta Paul Enferm. 2007;20(4):410-4.

18. Polit DF, Beck CT, Hungler BP. Fundamentos de pesquisa em enfermagem: métodos, avaliação e utilização. Porto Alegre: Artmed; 2004.

19. Kawamura S, Komori M. Smiling emphasizes perceived distinctiveness of faces. Percept Mot Skills. 2008;107(1):119-20.

20. Righart R, de Gelder B. Recognition of facial expressions is influenced by emotional scene gist. Cogn Affect Behav Neurosci. 2008;8(3):264-72.

21. Silva MJP. Análise comparativa da aplicação de um programa sobre comunicação não verbal para enfermeiros. Acta Paul Enferm. 2000;13(n.esp Pt 2):152-4.

22. Collins J. Education techniques for lifelong learning: principles of adult learning. Radiographica. 2004;25(5):1483-9. 